Espac. Saúde. 2021;22:e732

\title{
Tecnologias educacionais no cuidado às crianças com Diabetes Mellitus tipo 1: síntese do conhecimento
}

\author{
Educational technologies in the care of children with type 1 Diabetes \\ Mellitus: synthesis of knowledge
}

Tecnologías educativas en el cuidado de niños con Diabetes Mellitus tipo 1: sintesis del conocimiento

Jefferson Dantas da Costa ${ }^{1}$, Keila Maria de Azevedo Ponte Marques², Kairo

Cardoso da Frota ${ }^{3}$, Luciane Silva Oliveira ${ }^{4}$

1 Acadêmico de Enfermagem pela Universidade Estadual Vale do Acaraú. Sobral, Ceará

2 Enfermeira. Doutora em Cuidados Clínicos em Enfermagem e Saúde pela Universidade Estadual do Ceará (UECE). Docente do Curso de Graduação em Enfermagem da Universidade Estadual Vale do Acaraú. Sobral, Ceará.

3 Enfermeiro pela Universidade Estadual Vale do Acaraú Sobral, Ceará

4 Enfermeira pela Universidade Estadual Vale do Acaraú Sobral, Ceará

\section{RESUMO}

Objetivou-se identificar a síntese do conhecimento científico acerca da contribuição de tecnologias educacionais no cuidado às crianças com Diabetes Mellitus tipo 1. Trata-se de uma revisão integrativa realizada em junho e julho de 2020 nas bases de dados MEDLINE, LILACS e BDENF no período de 2015 a 2019. Após adotar os critérios de inclusão e exclusão 
ficaram oito artigos. As tecnologias educacionais utilizadas foram plataformas digitais, oficinas educacionais, simulações de casos, pôster e cartilha educacional. Identificou-se limitação de estudos com validação de tecnologias, que abordassem temáticas como atividades físicas, alimentação saudável e enfrentamento de situações psicossociais. Constatou-se a importância da atuação da equipe de enfermagem em intervenções de caráter educativo, realizadas de forma lúdica e criativa, incluindo pais e cuidador durante o processo.

Palavras-chave: Tecnologia Educacional. Diabetes Mellitus tipo 1. Enfermagem.

\begin{abstract}
This study aimed to identify the synthesis of scientific knowledge about the contribution of educational technologies in the care of children with type 1 Diabetes Mellitus. This is an integrative review conducted in June and July 2020 in the MEDLINE, LILACS, and BDENF databases of articles from 2015 to 2019. After adopting the inclusion and exclusion criteria, eight articles were selected. The educational technologies used were digital platforms, educational workshops, case simulations, poster, and educational booklet. We identified limitations of studies with validation of technologies that addressed themes such as physical activity, healthy eating, and coping with psychosocial situations. The importance of the nursing team's performance was identified in educational interventions run in a playful and creative way, including parents and caregivers during the process.
\end{abstract}

Keywords: Educational Technology. Diabetes Mellitus, Type 1. Nursing.

\title{
RESUMEN
}

Este estudio tuvo como objetivo identificar la síntesis de conocimientos científicos sobre la contribución de las tecnologías educativas en el cuidado de niños con Diabetes Mellitus tipo 1. Se trata de una revisión integradora realizada en junio y julio de 2020 en las bases de datos MEDLINE, LILACS y BDENF en el período 2015-2019. Tras adoptar los criterios de inclusión y exclusión, se adoptaron ocho artículos. Las tecnologías educativas utilizadas fueron plataformas digitales, talleres educativos, simulaciones de casos, póster y folleto educativo. Identificamos limitaciones de estudios con validación de tecnologías que abordaban temas como la actividad física, la alimentación saludable y el afrontamiento de situaciones psicosociales. Se verificó la importancia del desempeño del equipo de enfermería en las intervenciones educativas realizadas de una manera lúdica y creativa, incluyendo los padres y cuidadores durante el proceso.

Palabras clave: Tecnología Educacional. Diabetes Mellitus tipo 1. Enfermería. 


\section{INTRODUÇÃO}

A diabetes mellitus tipo 1 (DM1) é a principal causa de diabetes na infância. Estima-se que mais de 1 milhão de crianças e adolescentes vivem com a doença no mundo e as tendências revelam um aumento de 3\% a cada ano ${ }^{1}$. É preciso, porém, ir mais além e verificar que em países com carência de recursos médicos, as complicações metabólicas agudas da doença tendem a gerar maior mortalidade, devido, principalmente, à falta de insulina ${ }^{2}$.

Por outro lado, compreende-se que o acometimento por uma doença crônica na infância repercute na dinâmica familiar, na qual é preciso reformular o cotidiano para atender às necessidades de cuidado da criança, caracterizando-se como uma situação de vulnerabilidade em saúde ${ }^{3}$. No que diz respeito à DM1, destacam-se as dificuldades financeiras da família, as experiências negativas na escola relacionadas ao cuidado modificado, ao medo e à insegurança na gestão da insulinoterapia, dentre outras dificuldades ${ }^{4}$.

Nesse sentido, destaca-se como um problema emergente no contexto da saúde da criança, de evolução lenta e progressiva, que precisa de um tratamento especializado e de um acompanhamento longitudinal, no qual há a necessidade de um cuidado profissional apoiado, contínuo, proativo, ampliado e resolutivo. Daí a importância da utilização de estratégias educacionais para reduzir os riscos de complicações agudas e crônicas e de melhorar a qualidade de vida na infância ${ }^{5,6}$.

Nesse contexto, as Tecnologias Educacionais se inserem como meio oportuno, já que possuem como objetivo disseminar informações sobre demandas e necessidades existentes em diversos contextos sociais. Vale destacar, ainda, que elas visam potencializar a conscientização do sujeito em relação às decisões que permeiam o processo de viver ${ }^{7}$.

Nessa perspectiva, as tecnologias no âmbito educacional devem ser planejadas de acordo com o público-alvo, com a intenção de usá-las para possibilitar uma melhor interação com os participantes $^{8}$. Ademais, a sua utilização pode ser adequada aos diversos cenários, como hospital, escola, comunidade e domicílio, caracterizando-se como meio pedagógico para se comunicar com o paciente ${ }^{9}$.

A temática de educação em diabetes é recorrente aos profissionais de saúde que atuam em todos os níveis de atenção, principalmente ao enfermeiro da Atenção Primária à Saúde, pois durante sua formação acadêmica e, depois, em sua atuação profissional, é estimulado a desenvolver estratégias educacionais durante a consulta de enfermagem ${ }^{10}$.

Desse modo, este estudo se torna relevante ao possibilitar o conhecimento das Tecnologias Educacionais já desenvolvidas para a educação em saúde da criança com DM1, assim como ao evidenciar as principais lacunas existentes sobre a temática. Nessa perspectiva, objetivou-se identificar a produção de conhecimento científico sobre a contribuição das Tecnologias Educacionais no cuidado às crianças com DM1.

\section{MÉTODO}

Trata-se de uma revisão integrativa da literatura, realizada a partir das seguintes etapas: (1) Identificação do tema e definição da pergunta; (2) estabelecimento de critérios para inclusão e exclusão dos estudos; (3) definição das informações a serem extraídas dos estudos selecionados; (4) avaliação dos estudos; (5) interpretação dos resultados e (6) síntese do conhecimento ${ }^{11}$.

Para identificação do tema e definição da pergunta, foi utilizada a estratégia PICo (P- População: Crianças portadoras de Diabetes Mellitus tipo 1; I- Interesse: Tecnologia educacional; Cocontexto: Educação em saúde). Tendo, assim, como pergunta norteadora: "Qual a síntese do conhecimento científico acerca 
da contribuição de tecnologias educacionais para o cuidado das crianças com Diabetes Mellitus tipo 1?”.

A busca foi realizada nos meses de junho e julho de 2020, nas bases de dados: Medical Literature Analysis and Retrieval System Online MEDLINE/PubMed (via National Library of Medicine), Literatura Latino-Americana e do Caribe em Ciências da Saúde (LILACS) e Base de dados de Enfermagem (BDENF) por meio da Plataforma da Coordenação de Aperfeiçoamento de Pessoal de Nível Superior (CAPES). Foi realizada uma consulta nos Descritores em Ciências da Saúde (DECS)/ Medical Subject Headings (MeSH), em que se utilizou os seguintes descritores: Educação em Saúde / Health Education , Tecnologia Educacional/ Educational Technology e Diabetes Mellitus, associados ao operador boleano “AND” como estratégia de busca.

O cruzamento dos descritores obteve 3.809 publicações. Logo em seguida, iniciou-se a realização da segunda etapa da revisão, na qual foram adicionados os critérios de inclusão das tecnologias educacionais desenvolvidas com crianças de até 12 anos, artigos originais publicados de 2015 a 2019 em periódicos nacionais e internacionais nos idiomas inglês, português e espanhol, estando disponíveis gratuitamente para leitura na íntegra, alcançando 3.220 produções. Depois, foram inseridos os critérios de exclusão com textos duplicados, estudos não primários e que não respondessem à pergunta norteadora, restando, assim, 389 artigos. Selecionaram-se para leitura na íntegra, após a análise dos objetivos e resumos, 32 artigos. Por fim, foram elegíveis 08 artigos para compor esta revisão. Os estudos foram avaliados por dois pesquisadores independentes, nos quais as divergências foram sanadas por um terceiro revisor. Dessa forma, foi utilizada a estratégia Preferred Reporting Items for Systematic reviews and Meta-Analyses (PRISMA) ${ }^{12}$ - para seleção das publicações, pois os estudos se organizavam em quatro passos: identificação, seleção, elegibilidade e inclusão (Figura 1).

Figura 1 - Fluxograma do percurso de seleção dos estudos a partir das recomendações PRISMA. Sobral, Ceará, 2020.

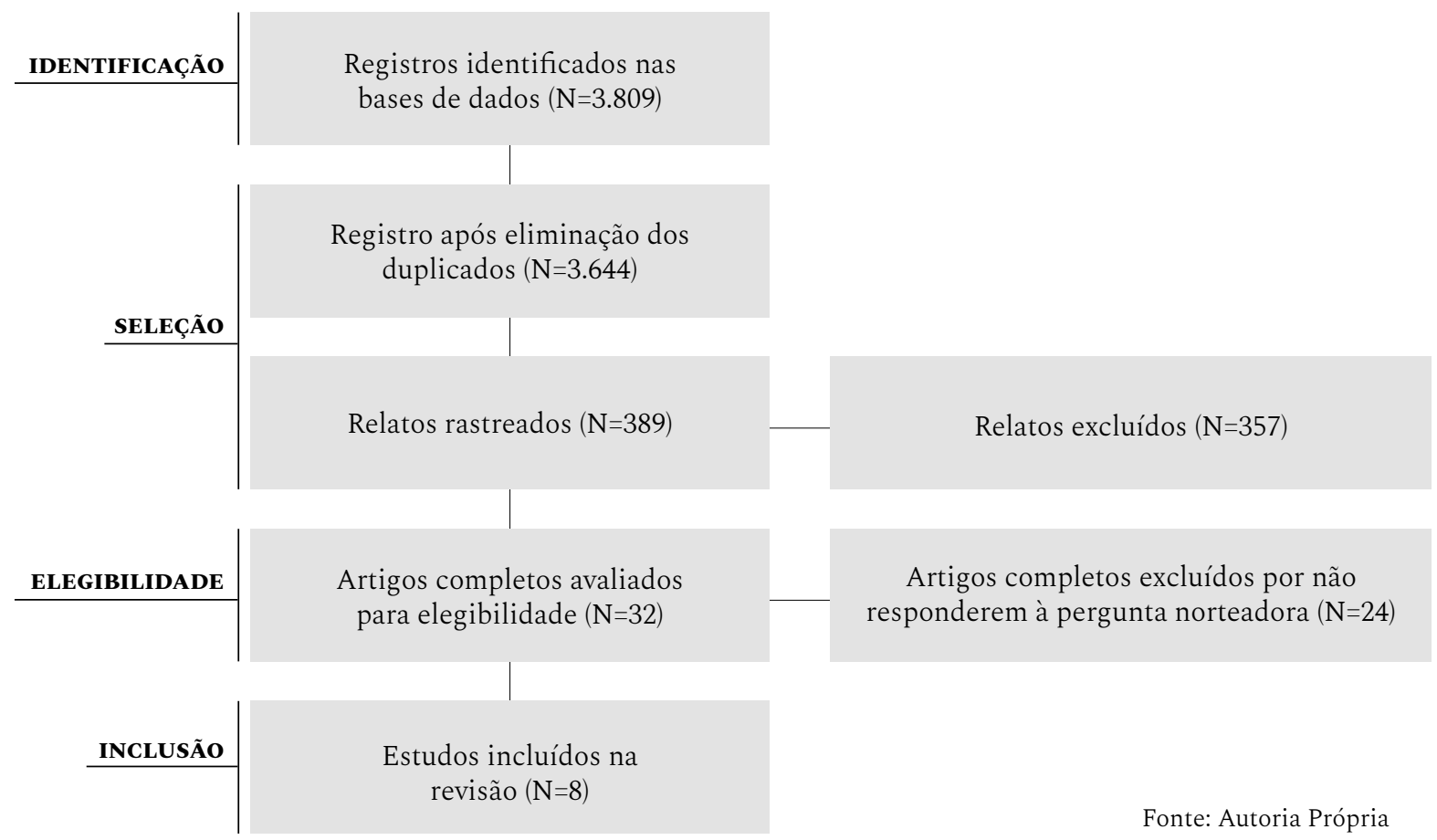


$\mathrm{Na}$ terceira etapa de definição das informações a serem extraídas dos estudos selecionados, os artigos incluídos foram analisados em uma planilha do Microsoft Office Excel $^{\oplus}$ 2016, agrupados e organizados pelos seguintes itens: título, objetivos, desenho do estudo, idioma, país, autores, ano, base de dados, periódico, tecnologia educacional, contribuição da tecnologia educacional e públicoalvo.

Dessa forma, foi possível realizar a quarta, quinta e sexta etapas, mediante avaliação, análise e interpretação dos artigos incluídos conforme literatura científica pertinente e atualizada na área para, assim, realizar a apresentação desta revisão.

\section{RESULTADOS}

A partir dos estudos analisados, constatou-se que seis foram encontrados na base de dados MEDLINE e dois na LILACS. Não foram encontrados estudos na BDENF. Desses, quatro estavam em periódico específico para cuidado à Diabetes Mellitus, três estavam em periódicos de enfermagem e um em outra revista da área da saúde.

Em relação ao ano de publicação: três em 2017; dois em 2018; um nos anos de 2015; um em 2016; um em 2019. Cinco artigos estavam no idioma inglês e quatro tinham como público-alvo crianças de 6 a 12 anos. Vale destacar que também foram incluídos os familiares e profissionais no cuidado à criança com Diabetes, bem como a população em geral. Com ênfase ao país de publicação, três ocorreram no Brasil e três nos Estados Unidos, seguidos de uma publicação no Canadá e uma na Nova Zelândia.

O quadro 1 apresenta os artigos incluídos na revisão integrativa de acordo com título, objetivo, base de dados, país de publicação e público-alvo.

Quadro 1 - Distribuição da produção científica sobre tecnologias educacionais em crianças com Diabetes. 2020 .

\begin{tabular}{|c|c|c|c|c|c|}
\hline $\mathbf{N}^{\circ}$ & Titulo & Objetivo & $\begin{array}{c}\text { Revista } \\
\text { Científica }\end{array}$ & $\begin{array}{c}\text { Base de } \\
\text { dados/País de } \\
\text { publicação }\end{array}$ & $\begin{array}{l}\text { Público- } \\
\text { alvo }\end{array}$ \\
\hline 1 & $\begin{array}{l}\text { Oficina educativa } \\
\text { baseada em atividades } \\
\text { lúdicas melhora o } \\
\text { automonitoramento } \\
\text { glicêmico entre crianças }{ }^{13} \text {. }\end{array}$ & $\begin{array}{l}\text { Avaliar a eficácia de uma } \\
\text { oficina educativa baseada em } \\
\text { atividades lúdicas para melhorar } \\
\text { a técnica de automonitoramento } \\
\text { glicêmico (AMG) de crianças } \\
\text { com diabetes tipo } 1\end{array}$ & $\begin{array}{l}\text { Revista Latino- } \\
\text { Americana de } \\
\text { Enfermagem }\end{array}$ & LILACS/Brasil & $\begin{array}{c}\text { Crianças } \\
\text { com idade } \\
\text { entre } 6 \text { e } 11 \\
\text { anos }\end{array}$ \\
\hline 2 & $\begin{array}{l}\text { Construção de cartilha } \\
\text { sobre insulinoterapia } \\
\text { para crianças com diabetes } \\
\text { mellitus tipo } 1^{14}\end{array}$ & $\begin{array}{l}\text { Descrever o processo de } \\
\text { construção de uma cartilha } \\
\text { educativa sobre insulinoterapia } \\
\text { para crianças com } \\
\text { diabetes mellitus tipo } 1 .\end{array}$ & $\begin{array}{l}\text { Revista } \\
\text { Brasileira de } \\
\text { Enfermagem }\end{array}$ & $\begin{array}{c}\text { MEDLINE/ } \\
\text { Brasil }\end{array}$ & $\begin{array}{l}\text { Crianças de } \\
8 \text { a } 11 \text { anos }\end{array}$ \\
\hline
\end{tabular}




\begin{tabular}{|c|c|c|c|c|c|}
\hline 3 & $\begin{array}{l}\text { Estrutura conceitual para } \\
\text { o desenvolvimento de } \\
\text { videogames para crianças } \\
\text { com diabetes mellitus tipo } \\
1^{15}\end{array}$ & $\begin{array}{l}\text { Apresentar uma estrutura } \\
\text { teórica conceitual para o } \\
\text { desenvolvimento de videogames } \\
\text { para crianças com diabetes } \\
\text { mellitus tipo } 1 .\end{array}$ & $\begin{array}{l}\text { Revista Latino- } \\
\text { Americana de } \\
\text { Enfermagem }\end{array}$ & $\begin{array}{l}\text { LILACS / } \\
\text { Brasil }\end{array}$ & $\begin{array}{c}\text { Crianças } \\
\text { entre } 7 \text { e } 12 \\
\text { anos }\end{array}$ \\
\hline 4 & $\begin{array}{l}\text { CDE Perspectives } \\
\text { of Providing New- } \\
\text { Onset Type } 1 \text { Diabetes } \\
\text { Education using } \\
\text { Formal Vignettes and } \\
\text { Simulation }^{16}\end{array}$ & $\begin{array}{l}\text { O objetivo deste artigo é } \\
\text { descrever as perspectivas } \\
\text { de educadores de diabetes } \\
\text { certificados (CDEs) de ensinar } \\
\text { aos pais de crianças com diabetes } \\
\text { mellitus recém-diagnosticado } \\
\text { tipo } 1 \text { (T1DM) habilidades de } \\
\text { gerenciamento precoce de } \\
\text { diabetes usando estratégias de } \\
\text { ensino formais e uma simulação } \\
\text { de paciente humano (HPS) para } \\
\text { aumentar/aprimorar o processo } \\
\text { de ensino-aprendizagem. }\end{array}$ & $\begin{array}{l}\text { Diabetes } \\
\text { Education }\end{array}$ & $\begin{array}{l}\text { MEDLINE/ } \\
\text { Estados } \\
\text { Unidos }\end{array}$ & $\begin{array}{l}\text { Pais de } \\
\text { crianças } \\
\text { menores de } \\
13 \text { anos }\end{array}$ \\
\hline 5 & $\begin{array}{l}\text { A brief campaign } \\
\text { to prevent diabetic } \\
\text { ketoacidosis in children } \\
\text { newly diagnosed with } \\
\text { type } 1 \text { diabetes mellitus: } \\
\text { The NO-DKA Study }{ }^{17}\end{array}$ & $\begin{array}{l}\text { Avaliar se uma campanha } \\
\text { educacional simples e } \\
\text { facilmente entregue poderia } \\
\text { reduzir o risco de cetoacidose } \\
\text { diabética de início novo. }\end{array}$ & $\begin{array}{l}\text { Pediatric } \\
\text { Diabetes }\end{array}$ & $\begin{array}{c}\text { MEDLINE/ } \\
\text { Nova Zelândia }\end{array}$ & $\begin{array}{l}\text { População } \\
\text { geral da } \\
\text { região }\end{array}$ \\
\hline 6 & $\begin{array}{l}\text { Grandparent Education } \\
\text { Through Simulation } \\
\text { Diabetes }^{18}\end{array}$ & $\begin{array}{l}\text { O objetivo deste estudo foi } \\
\text { explorar a viabilidade do uso da } \\
\text { simulação de pacientes humanos } \\
\text { (HPS) para ensinar a gestão do } \\
\text { diabetes tipo 1 (T1DM) aos avós } \\
\text { de netos com T1DM. }\end{array}$ & $\begin{array}{l}\text { The Diabetes } \\
\text { EDUCATOR }\end{array}$ & $\begin{array}{l}\text { MEDLINE/ } \\
\text { Estados } \\
\text { Unidos }\end{array}$ & $\begin{array}{l}\text { Avós de } \\
\text { crianças } \\
\text { menores de } \\
12 \text { anos com } \\
\text { T1DM. }\end{array}$ \\
\hline 7 & $\begin{array}{l}\text { Evaluation of a } \\
\text { Computerized Self- } \\
\text { Management Tool for } \\
\text { Children with Type } 1 \\
\text { Diabetes: A Pilot Project }{ }^{19}\end{array}$ & $\begin{array}{l}\text { Avaliar se o SMS KiDi é uma } \\
\text { ferramenta eficaz para motivar } \\
\text { as crianças a automonitorar } \\
\text { seus níveis de glicose no sangue } \\
\text { diariamente. }\end{array}$ & $\begin{array}{l}\text { Canadian } \\
\text { Journal of } \\
\text { Diabetes }\end{array}$ & $\begin{array}{c}\text { MEDLINE/ } \\
\text { Canadá }\end{array}$ & $\begin{array}{c}\text { Crianças } \\
\text { entre } 8 \text { e } 12 \\
\text { anos. }\end{array}$ \\
\hline 8 & $\begin{array}{l}\text { Improving paediatric } \\
\text { residents' knowledge and } \\
\text { perspectives regarding } \\
\text { the insulin pump using } \\
\text { a novel educational } \\
\text { workshop }{ }^{20}\end{array}$ & $\begin{array}{l}\text { Investigar os conhecimentos } \\
\text { e percepções dos residentes } \\
\text { pediátricos categóricos (RES) } \\
\text { em nossa instituição em relação } \\
\text { às bombas de insulina (IPs) e } \\
\text { o impacto após uma oficina } \\
\text { direcionada. }\end{array}$ & Postgrad Med J & $\begin{array}{l}\text { MEDLINE/ } \\
\text { Estados } \\
\text { Unidos }\end{array}$ & Residentes \\
\hline
\end{tabular}


Quadro 2 - Distribuição da produção científica sobre tecnologias educacionais em crianças com Diabetes. 2020.

\begin{tabular}{|c|c|c|c|}
\hline $\mathbf{N}^{\circ}$ & Autor/ano & $\begin{array}{l}\text { Tecnologia } \\
\text { educacional }\end{array}$ & Contribuições da tecnologia \\
\hline 1 & Kaneto et al (2018) & Oficina educativa & $\begin{array}{l}\text { A oficina demonstrou-se útil na melhora da conformidade } \\
\text { da técnica de automonitoramento glicêmico. }\end{array}$ \\
\hline 2 & Moura et al (2017) & Cartilha educativa & $\begin{array}{l}\text { A construção da cartilha: Aplicando a insulina: a aventura } \\
\text { de Beto, figurou-se como proposta para favorecer o } \\
\text { empoderamento e promover a saúde de crianças com } \\
\text { Diabetes Mellitus tipo } 1 .\end{array}$ \\
\hline 3 & Sparapani et al (2019) & Videogame educativo & $\begin{array}{l}\text { Contribuem para o avanço nas discussões de como as } \\
\text { teorias comportamentais e seus determinantes devem } \\
\text { estar relacionados ao desenho de videogames criativos e } \\
\text { divertidos, considerando o perfil da população-alvo, assim } \\
\text { como suas necessidades e preferências. }\end{array}$ \\
\hline 4 & $\begin{array}{l}\text { Ramchandani et al } \\
\text { (2016) }\end{array}$ & $\begin{array}{l}\text { Ensino e Simulação } \\
\text { com uso de manequim }\end{array}$ & $\begin{array}{l}\text { Essas metodologias podem ser usadas com sucesso para } \\
\text { educar pais de crianças com DM1 de início novo sobre } \\
\text { hipoglicemia, hiperglicemia e gerenciamento de padrões, } \\
\text { além de serem preferidas pelos educadores. }\end{array}$ \\
\hline 5 & Derraik et al (2018) & Pôster & $\begin{array}{l}\text { Uma campanha não direcionada para aumentar a } \\
\text { conscientização sobre os sintomas do diabetes na } \\
\text { juventude levou apenas alguns cuidadores a procurar } \\
\text { atendimento médico oportuno, tendo impacto limitado. }\end{array}$ \\
\hline 6 & $\begin{array}{l}\text { Maguire, Crawford, } \\
\text { Sullivan-Bolyai (2015) }\end{array}$ & Simulação-Diabetes & $\begin{array}{l}\text { Este estudo demonstrou a viabilidade de recrutar e } \\
\text { reter avós em um ensaio clínico usando uma simulação } \\
\text { de intervenção para ensinar a gestão DM1. Após } \\
\text { a intervenção, todas as pontuações dos avós para } \\
\text { conhecimento, confiança e medo do DM1 mostraram } \\
\text { melhora significativa. }\end{array}$ \\
\hline 7 & Dyal et al (2017) & $\begin{array}{l}\text { Plataforma digital - } \\
\text { SMS KiDi }\end{array}$ & $\begin{array}{l}\text { KiDi SMS motivou } 85 \% \text { de seus usuários a autogerenciar } \\
\text { suas leituras de glicemia diariamente. }\end{array}$ \\
\hline 8 & Bansal et al (2018) & Oficina educacional & $\begin{array}{l}\text { Intervenções educativas são necessárias para familiarizar } \\
\text { os futuros médicos com IPs para permitir que os hospitais } \\
\text { forneçam seu uso sistemático e seguro. }\end{array}$ \\
\hline
\end{tabular}

Fonte: Autoria própria

\section{DISCUSSÃo}

A amostra final dos artigos apresentou uma variedade de TE desenvolvidas para o cuidado à criança com Diabetes Mellitus tipo 1, revelando o empenho de pesquisadores e profissionais da saúde em produzir atividades de educação ao público-alvo. Sendo assim, as tecnologias educacionais elucidadas nos artigos foram plataformas digitais, oficinas educacionais, simulação de casos, pôster e cartilha educacional. 
Esses resultados convergem com as atuais produções de TE voltadas para crianças com doenças crônicas e seus familiares, as quais geralmente envolvem o uso da informática, material de mídia ou audiovisual, cartilhas, manuais, histórias infantis e jogo educativo ${ }^{21}$. Métodos que têm o intuito de gerar o empoderamento da criança no autocuidado, com a consequente construção de independência e autonomia com o passar dos anos ${ }^{14}$.

Está claro que as TE devem ser criativas e dinâmicas, daí as plataformas digitais surgem como um dos meios para atingir esse objetivo. Assim, são eficientes ao passo em que agregam recursos visuais e auditivos para estimular o indivíduo a conhecer a temática abordada. O problema, entretanto, é o acesso à tecnologia da informação, visto que nem todo mundo possui à sua disposição internet e/ou aparelho digital ${ }^{19,21}$.

Com base nesse ponto, os materiais impressos se tornam uma alternativa didática com grande potencial, pois são de fácil acesso pelas pessoas de diversos níveis socioeconômicos. Além disso, quando construídos com uma leitura atrativa e um design inovador, tendem a potencializar o processo de ensino-aprendizagem ${ }^{14}$.

Um estudo ${ }^{14}$ realizado com 17 crianças, na faixa etária de 8 a 12 anos, revelou a eficiência de uma cartilha educativa no conhecimento e no autocuidado sobre insulinoterapia de crianças com DM1. Em contrapartida, vale também destacar uma pesquisa ${ }^{17}$ realizada na nova Zelândia que obteve um impacto bastante limitado, na qual divulgou pôsteres educacionais sobre as características diagnósticas de crianças com início de DM1, mas que apenas 2,17\% dos novos casos, em dois anos de pesquisa, relataram ter conhecimento sobre a estratégia educacional aplicada.

Não menos importante que as TE abordadas, são os programas educacionais que envolvem o uso de simulação de casos. Conforme verificado pelos estudos $^{16,18}$, essa metodologia que já é usada para o ensino de estudantes e profissionais da área da saúde, também se mostraram eficientes para educação de pais e avós de crianças com DM1, melhorando significativamente o medo, conhecimento e confiança sobre a doença.

Quanto ao conteúdo das tecnologias educacionais, as temáticas abordadas foram monitoramento glicêmico, resolução de problemas relacionados a complicações agudas, resolução de riscos de complicações crônicas e uso da medicação insulina. Dessa forma, ao levar em consideração os 7 padrões de educação em diabetes destacados pela American Association of Diabetes Educators $^{22}$, há uma deficiência na produção de TE relacionadas à atividade física, alimentação saudável e enfrentamento saudável de possíveis problemas psicossociais.

É importante dar ênfase a essas nuances que envolvem o cuidado integral às crianças com DM1, quanto à alimentação saudável há barreiras comuns encontradas pelas famílias. Vale destacar as preferências alimentares da criança, o preço dos alimentos adequados e o tempo para planejar e realizar as compras ${ }^{23}$. Em uma pesquisa realizada nos Estados Unidos, a diferença entre uma lista de compras de alimentos mais saudáveis e uma lista de compras padrão, fica em torno de $18 \%$ mais cara ${ }^{24}$. Daí a importância de serem desenvolvidas tecnologias educacionais que abordem além do ensino sobre a doença, também as variáveis socioeconômicas da região e do público-alvo da intervenção.

Verificou-se o predomínio de publicações voltadas diretamente às crianças, mas também houve estudos que incluíram os familiares ${ }^{16,18}$, como pais e avós, profissionais de saúde ${ }^{20}$ e o público em geral ${ }^{17}$ no processo educacional sobre DM1. Sob essa ótica, ganha particular relevância a forma de ensino a depender da faixa etária da criança, dado que um estudo $^{25}$ destaca a divisão das responsabilidades da seguinte forma: de 0 a 2 anos, pais com gestão total do diabetes, contam apenas com a cooperação das crianças; de 3 a 5 anos, pais com gestão total do 
diabetes com rara responsabilidade dada à criança; De 6 a 12 anos, mais responsabilidade dada à criança, contando sempre com a supervisão dos pais, como contagem de carboidrato, verificação da glicemia para exercício, dentre outras ${ }^{26}$. Esse fato justifica o porquê das Tecnologias educacionais desse estudo, quando realizadas com as crianças, serem direcionadas a uma faixa etária de 6 a 12 anos, momento no qual há maior possibilidade de autogerenciamento da DM1.

Vale destacar evidências de que o controle glicêmico está relacionado ao desempenho escolar, no qual as crianças que possuem menor controle metabólico apresentam notas mais baixas em relação à população em geral, além de também terem maior índice de faltas/ausência escolar ${ }^{27,28}$. Daí surge a importância da educação em saúde ocorrer não apenas no momento do diagnóstico, mas também de forma contínua acompanhando o desenvolvimento da criança e, para isso, também deve ser realizada educação aos profissionais de saúde ${ }^{29}$.

É preciso, porém, ir mais além e verificar que a equipe de enfermagem atua em diversos cenários nos quais há atendimento pediátrico, como na atenção primária, clínicas ambulatoriais, urgência e emergência, entre outras ${ }^{13}$. Além disso, verificouse 5 estudos ${ }^{13-16,18}$ nessa pesquisa nos quais a construção e aplicação das tecnologias educacionais foram realizadas por enfermeiros, sendo que dois desses possuem o título de Educador de Diabetes Certificado (CDE) dos Estados Unidos, evidenciando o empenho da profissão em produzir e aplicar métodos de educação em saúde eficientes, criativos e dinâmicos. Apesar disso, é importante enfatizar a importância da realização da educação permanente para aperfeiçoar as práticas de educação em saúde por meio de TE, tendo em vista a qualificação e aperfeiçoamento da assistência de enfermagem ${ }^{30}$.

Nessa perspectiva, torna-se oportuno contextualizar o processo de validação metodológico das tecnologias educacionais, que consiste na qualificação e potencialização de uma nova intervenção ou de uma já existente e leva em consideração aspectos estruturais, conceituais e organizacionais ${ }^{7,31}$. Neste estudo, nenhuma das tecnologias educacionais realizou validação por meio da participação de juízes-especialistas sobre a temática, porém foi realizada uma escuta sensível dos participantes.

\section{CONCLUSÕES}

A síntese do conhecimento mostrou que as tecnologias educativas voltadas a crianças com Diabetes Mellitus usaram plataformas digitais, oficinas educacionais, simulação de casos, pôster e cartilha educacional como estratégias que contribuem para o cuidado às crianças com DM 1, estando associadas ao caráter educativo de orientar de forma lúdica e criativa, incluindo também pais e cuidadores.

Pode-se verificar que a produção do conhecimento na área esteve muito atrelada ao contexto dos cuidados de enfermagem, os quais possuem características que vão de encontro às necessidades das famílias e das crianças que vivenciam o processo de adoecimento por DM 1. Trata-se de cuidados sistematizados, individualizados, comprometidos com as reais necessidades, sendo as tecnologias educacionais estratégias que se enquadram nessas especificidades.

A principal limitação deste estudo foi o acesso gratuito a artigos sobre a temática, tendo em vista que havia produções que se enquadravam nos critérios de inclusão, porém com acesso limitado a assinantes do periódico. Apesar disso, espera-se que esta revisão estimule a produção de conhecimento acerca do uso das tecnologias educacionais no cuidado à criança com DM 1, a partir de investigações experimentais.

\section{REFERÊNCIAS}

1. IDF - International Diabetes Federation. IDF Diabetes Atlas. 9ed.,2019. Disponível em http://www.diabetesatlas. org. 
2. SBD - Sociedade Brasileira de Diabetes. Diretrizes da Sociedade Brasileira de Diabetes: 2019-2020. São Paulo: Clannad; 2019.

3. Pinto MMPS, Coutinho SED, Collet N. Doença crônica na infância e a atenção dos serviços de saúde. Ciênc Cuid Saúde [Internet]. 2016 [Acesso em 2020 jun 01];15(3):498506. Disponível em: https://pesquisa.bvsalud.org/portal/resource/pt/biblio-974873

4. Okido ACC, Almeida A, Vieira MM, Neves ET, Mello DF, Lima RAG. As demandas de cuidado das crianças com Diabetes Mellitus tipo 1. Esc. Anna Nery [Internet]. 2017 [Acesso em 2020 jun 01];21(2):e20170034. Disponível em: https://www.scielo.br/pdf/ean/v21n2/1414-8145-ean-2102-e20170034.pdf

5. Hermes TSV, Viera CS, Rodrigues RM, Toso BRGO, Fonseca LMM. Criança diabética do tipo 1 e o convívio familiar: repercussões no manejo da doença. Saúde debate [Internet]. 2018 [Acesso em 2020 jun 05];42(119):927-939. Disponível em: https://www.scielo.br/scielo.php?script=sci_arttext\&pid=S0103-11042018000400927

6. Nóbrega VM, Silva MEA, Fernandes LTB, Viera CS, Reichert APS, Collet N. Doença crônica na infância e adolescência: continuidade do cuidado na Rede de Atenção à Saúde. Rev Esc Enferm. USP [Internet]. 2017 (Acesso em 2020 jun 05] ;51:e03226.Disponível em: https://www.scielo. br/pdf/reeusp/v51/pt_1980-220X-reeusp-51-e03226.pdf

7. Teixeira E, Mota VMSS. Educação em saúde: tecnologias educacionais em foco. $1^{\circ}$ ed. São Paulo. Difusão: 2011.

8. Silva DML, Correiro FA, Mello R. Tecnologias educacionais na assistência de enfermagem em educação em saúde: revisão integrativa. Rev Enferm UFPE [Internet]. 2017 [Acesso em 2020 jun 05];11(Supl. 2):10441051. Disponível em: https://pesquisa.bvsalud.org/portal/ resource/pt/bde-30877

9. Áfio ACE, Balbino AC, Alves MDS, Carvalho LV, Santos MCL, Oliveira NR. Análise do conceito de tecnologia educacional em enfermagem aplicada ao paciente. Rev RENE [Internet]. 2014 [Acesso em 2020 jun 05];15(1);158165. Disponível em: http://www.periodicos.ufc.br/rene/ article/view/3108

10. Moreira TMM, Pinheiro JAM, Florêncio RS, Cestari VRF. Tecnologias para a promoção e o cuidado em saúde. $1^{\circ}$ ed. Fortaleza. EdUECE:2018.

11. Mendes KDS, Silveira RCCP, Galvão CM. Revisão integrativa: método de pesquisa para a incorporação de evidências na saúde e na enfermagem. Texto contexto - enferm. [Internet]. 2008 Dec [Acesso em 2020 jun 08];17(4):758-764. Disponível em: https://www.scielo.br/ scielo.php?pid=s0104-07072008000400018\&script $=$ sci_ abstract\&tlng=pt

12. Moher D, Liberati A, Tetzlaff J, Altman DG. The PRISMA Group. Preferred Reporting Items for Systematic Reviews and Meta-Analyses: The PRISMA Statement. [Internet]. 2009 [Acesso em 2020 jun 08];6:e1000097. Disponível em: www.prisma-statement.org.

13. Kaneto LA, Damião EBC, Verissimo MLÓR, Rossato LM, Toriyama ATM, Szylit R. Oficina educativa baseada em atividades lúdicas melhora o automonitoramento glicêmico entre crianças. Rev Latino-Am Enferm [Internet]. 2018 [Acesso em 2020 ago 12]; 26:e3039. Disponível em: https://www.scielo.br/pdf/rlae/v26/pt_0104-1169-rlae26-e3039.pdf

14. Moura DJM, Moura NS, Menezes LCG, Barros AA, Guedes MVC. Construção de cartilha sobre insulinoterapia para crianças com diabetes mellitus tipo 1. Rev Bras Enferm. [Internet]. 2017 [Acesso em 2020 ago 12];70(1):7-14. Disponível em: https://www.scielo.br/ scielo.php?pid=S0034-71672017000100007\&script $=$ sci_ abstract\&tlng=pt

15. Sparapani VC, Fels S, Kamal N, Nascimento LC. Estrutura conceitual para o desenvolvimento de videogames para crianças com diabetes mellitus tipo 1. Rev Latino-Am Enferm [Internet]. 2019 [Acesso em 2020 ago 12];27:e3090. Disponível em: https://www.scielo.br/scielo. php?script=sci_arttext\&pid=S0104-11692019000100315\&ln $\mathrm{g}=\mathrm{en} \& \mathrm{nrm}=\mathrm{iso} \& \mathrm{t} \operatorname{lng}=\mathrm{pt}$

16. Ramchandani $\mathrm{N}$, Johnson $\mathrm{K}$, Cullen $\mathrm{K}$, Hamm $\mathrm{T}$, Bisordi J, Sullivan-Bolyai S. CDE Perspectives of Providing New-Onset Type 1 Diabetes Education Using Formal Vignettes and Simulation. Diabetes Educ. [Internet] 2017 [Acesso em 2020 ago 15];43(1):97-104. Disponível em: https://journals.sagepub.com/doi/10.1177/01457217166768 93?url_ver=Z39.88-2003\&rfr_id=ori:rid:crossref.org\&rfr_ dat $=$ cr_pub $\% 20 \% 200$ pubmed

17. Derraik JGB, Cutfield WS, Maessen SE, Hofman PL, Kenealy T, Gunn AJ, et al. A brief campaign to prevent diabetic ketoacidosis in children newly diagnosed with type 1 diabetes mellitus: The NO-DKA Study. Pediatr Diabetes. [Internet] 2018 [Acesso em 2020 ago 15];19(7):12571262. Disponível em: https://onlinelibrary.wiley.com/doi/ abs/10.1111/pedi.12722 
18. Maguire LL, Crawford S, Sullivan-Bolyai S. Grandparent Education Through Simulation-Diabetes. Diabetes Educ. [Internet] 2015 [Acesso em 2020 ago 22];41(6):678689. Disponível em: https://journals.sagepub.com/doi/ 10.1177/0145721715607982?url_ver=Z39.88-2003\&rfr_ id=ori:rid:crossref.org\&rfr_dat=cr_pub\%20\%200pubmed

19. Dyal N, McAssey K, Agarwal G. Evaluation of a Computerized Self-Management Tool for Children with Type 1 Diabetes: A Pilot Project. Can J Diabetes. [Internet] 2017 [Acesso em 2020 ago 22];41(2):124-128. Disponível em: https://www.canadianjournalofdiabetes.com/article/S14992671(16)30643-8/fulltext

20. Bansal S, Marwa A, Kasturi K, Perez-Colon S. Improving paediatric residents' knowledge and perspectives regarding the insulin pump using a novel educational workshop. Postgrad Med J. [Internet] 2018 [Acesso em 2020 ago 25];94(1108):87-91. Disponível em: https://pmj.bmj.com/ content/94/1108/87.long

21. Costa C, Pacheco S, Soeiro G, Adame D, Peres P, Araújo B. Construção e validação de materiais educativos para criança com doença crônica: uma revisão integrativa. Rev Enferm UERJ. [Internet] 2018 [Acesso em 2020 ago 25];26: e34208. Disponível em: https://www.e-publicacoes.uerj.br/ index.php/enfermagemuerj/article/view/34208

22. American Association of Diabetes Educators. Diabetes education curriculum: a guide to successful self-management. Quick Overview. Chicago: AADE; 2015.

23. Patton SR, Clements MA, George K, Goggin K. "I Don't Want Them to Feel Different": A Mixed Methods Study of Parents' Beliefs and Dietary Management Strategies for Their Young Children with Type 1 Diabetes Mellitus. J Acad Nutr Diet. [Internet] 2016 [Acesso em 2020 set 01];116(2):272282. Disponível em: https://www.sciencedirect.com/ science/article/abs/pii/S2212267215011119

24. Patton SR, Goggin K, Clements MA. The Cost of a Healthier Diet for Young Children With Type 1 Diabetes Mellitus. J Nutr Educ Behav. [Internet] 2015 [Acesso em 01 set 2020];47(4):361-6.e1. Disponível em: https://www.ncbi. nlm.nih.gov/pmc/articles/PMC4509633/

25. Markowitz JT, Garvey KC, Laffel LM. Developmental changes in the roles of patients and families in type 1 diabetes management. Curr Diabetes Rev. [Internet] 2015
[Acesso em 2020 set 10];11(4):231-238. Disponível em: https://www.ncbi.nlm.nih.gov/pmc/articles/PMC4826732/.

26. Iovane B, Cangelosi AM, Bonaccini I, Mauro DD, Scarabello C, Panigari A, et al. Diabetic ketoacidosis at the onset of Type 1 diabetes in young children Is it time to launch a tailored campaign for DKA prevention in children $<5$ years?. Acta Biomed. [Internet] 2018 [Acesso em 2020 set 10];89(1):67-71. Disponível em: https://www.ncbi.nlm.nih. gov/pmc/articles/PMC6357617/.

27. Román R, Garrido V, Novoa V, Mundaca G, Pichuante E, Rivera A, et al. Control metabólico y rendimiento escolar en niños con diabetes tipo 1 [Metabolic control and school performance in children with type 1 diabetes]. Rev Chil Pediatr. [Internet] 2017 [Acesso em 2020 set 10];88(5):586594.Disponível em: https://scielo.conicyt.cl/scielo. php?script=sci_arttext\&pid=S0370-41062017000500003.

28. Fleming M, Fitton CA, Steiner MFC, McLay JS, Clark D, King A et al. Educational and Health Outcomes of Children Treated for Type 1 Diabetes: Scotland-Wide Record Linkage Study of 766,047 Children. Diabetes Care. [Internet] 2019 [Acesso em 2020 set 15];42(9):1700-1707. Disponível em: https://care.diabetesjournals.org/content/ early/2019/07/05/dc18-2423

29. Beran D, Golay A. Initial versus ongoing education: Perspectives of people with type 1 diabetes in 13 countries. Patient Educ Couns. [Internet] 2017 [Acesso em 2020 set 15];100(5):1012-1018. Disponível em: https://www.sciencedirect.com/science/article/abs/pii/ S0738399116305717

30. Santos RR, Zagonel IPS, Sanches LC, Ribeiro ER, Garbelini MCL. Educação em saúde: Conhecimento dos enfermeiros para prevenção da lesão por pressão no domicílio. Esp Saúde (Online). [Internet] 2018 [Acesso em 2020 set 15];19(9):54-63. Disponível em: http:// docs.bvsalud.org/biblioref/2019/03/981816/5-educacao-emsaude_612-1070-1-ed2.pdf

31. Rocha EP, Oliveira APP, Esteveres AVF. Validação das tecnologias educacionais na área de Enfermagem: uma revisão integrativa. Scientia Amazonia. [Internet] 2015 [Acesso em 2020 ago 01];4(3):41-47. Disponível em: http:// scientia-amazonia.org/wp-content/uploads/2016/06/v4-n341-47-2015.pdf 